\title{
La photoémission VUV résolue en temps sur des solides isolants
}

\author{
G. Geoffroy, S. Guizard, A. Belsky ${ }^{1}$, E. Constant ${ }^{1}$, P. Martin ${ }^{1}$ et E. Mével ${ }^{1}$ \\ Laboratoire des Solides Irradiés, CEA/DSM/DRECAM-CNRS, École Polytechnique, \\ 91128 Palaiseau cedex, France \\ ${ }^{1}$ Laboratoire CELIA, Université de Bordeaux l et CNRS, 351 cours de la Libération, \\ 33405 Talence, France
}

\begin{abstract}
Résumé : L'objectif de ce travail expérimental est l'étude de la dynamique des processus de relaxations électroniques dans les solides, et en particulier dans les diélectriques. La technique la plus couramment employée pour étudier ces phénomènes est la photoémission résolue en temps, à l'aide d'impulsions laser ultra-brèves. Une première impulsion excite les électrons da solide, et une seconde impulsion provoque l'ionisation d'une partie de ces électrons. La mesure de l'énergie cinétique de ces photoélectrons en fonction du retard entre les impulsions pompe et sonde donne une image directe de l'évolution de leur distribution énergétique. Cette technique très puissante a déjà été utilisée pour étudier les cinétiques de relaxation dans les semi-conducteurs et les métaux. En effet les énergies de photons nécessaires aux excitations électroniques correspondent aux longueurs d'onde d'émission des lasers femtosecondes les plus courants. Les harmoniques d'ordres élevés obtenues à partir de lasers femtosecondes intenses permettent d'étendre ces études à des matériaux isolants à grande bande interdite. Les résultats d'une étude systématique de la relaxation d'électrons de grande énergie $(\cong 30 \mathrm{eV})$ dans différents diélectriques $\left(\mathrm{SiO}_{2}, \mathrm{CeF}_{3}\right)$, réalisée avec la source d'harmoniques du laboratoire CELIA sont comparés et discutés.
\end{abstract}

\section{INTRODUCTION}

Les sources de rayonnement UV et X générées à partir de lasers intenses ont fait l'objet d'un travail de recherche important et deviennent un outil de plus en plus utilisé en physique fondamentale. Les harmoniques d'ordres élevés, impulsions de photons cohérents, naturellement synchrones et d'une durée égale ou inférieure à celles du laser qui les a générées, sont en particulier parfaitement adaptées aux expériences résolues en temps dans un domaine d'énergie (de 10 à $50 \mathrm{eV}$ ) encore peu exploré. En physique des solides, elles ont été utilisées par exemple comme sonde d'une modification de la distribution électronique induite par un faisceau visible dans les semi-conducteurs [1], où une modification de l'oxydation de la surface d'un échantillon d'argent [2]. Il s'agit dans ces cas de spectroscopie de photoémission ultraviolette (UPS) «traditionnelle», mais résolue en temps. Dans les expériences présentées ici, nous utilisons le faisceau harmonique dans une expérience de photoémission à deux couleurs, comme pompe, avec pour objectif la mesure de la relaxation des électrons excités dans un solide avec une énergie importante - de 13 à $30 \mathrm{eV}$. Au cours de ce type d'expérience le solide isolant à large bande interdite (7 à $10 \mathrm{eV})$ est excité par un faisceau VUV ayant une énergie jusqu'à 3 à 4 fois celle de la bande interdite. Lorsque le faisceau IR $(1.57 \mathrm{eV})$ arrive sur le solide déjà excité par le faisceau harmonique une partie des électrons absorbe encore un photon et quitte le solide avec une plus grande énergie cinétique. L'évolution du spectre de photoélectrons en fonction du retard entre les deux impulsions donne accès à la dynamique de relaxation des électrons excités. 


\section{EXPERIENCE : PRINCIPE ET RESULTATS}

Les expériences ont été réalisées au laboratoire CELIA qui dispose d'un laser Titane-Saphir $(800 \mathrm{~nm})$ délivrant à la cadence de $1 \mathrm{KHz}$ des impulsions d'une durée de 45 fs et d'une énergie de $4 \mathrm{~mJ}$. Une partie de ce faisceau sert à générer les harmoniques d'ordres élevés dans une fibre de silice ou s'écoule un gaz rare - Argon en général. L'harmonique souhaitée est sélectionnée au moyen d'un monochromateur comprenant un miroir torique de focale $2 \mathrm{~m}$ et un réseau, et une fente de sortie placée à l'entrée de l'enceinte d'expérience. Ce système permet de sélectionner une harmonique quelconque, au détriment de la résolution temporelle, l'élargissement induit par le réseau est de l'ordre de $600 \mathrm{fs}$. Le faisceau sonde est constitué du faisceau IR résiduel, avec un délai contrôlé par une ligne à retard, et focalisé par une lentille de 3 mètres de focale. L'énergie de ce faisceau sonde varie de $40 \mu \mathrm{J}$ à $190 \mu \mathrm{J}$ pour un diamètre à mi- intensité de $350 \mu \mathrm{m}$. Les deux faisceaux sont incidents à $45^{\circ}$ sur l'échantillon, placé dans une enceinte à ultravide $\left(10^{-9} \mathrm{mbar}\right)$ perpendiculairement au détecteur d'électrons un analyseur hémisphérique commercial (CLAM 4 de la marque VG Microtech). Afin de s'affranchir du problème délicat de charge de surface les échantillons sont chauffés entre $200^{\circ} \mathrm{C}$ (échantillons $\mathrm{CsI}$ et $\mathrm{CeF}_{3}$ ) et $400^{\circ} \mathrm{C}$ (échantillons $\mathrm{SiO}_{2}$ et $\mathrm{Al}_{2} \mathrm{O}_{3}$ ). Pour les

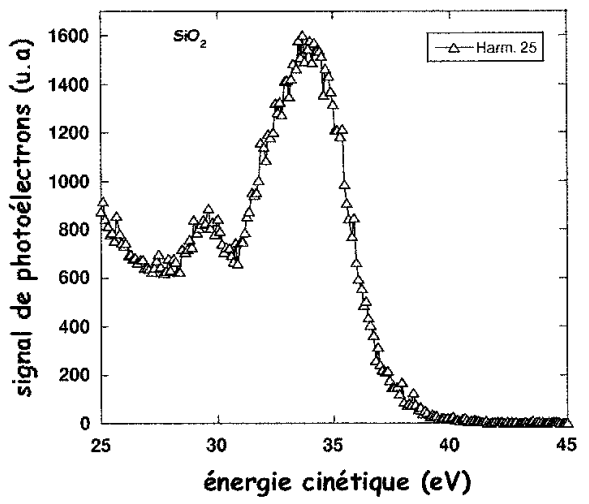

Figurel: spectre de photoémission obtenus sur le $\mathrm{SiO}_{2}$ avec le faisceau harmonique de $39.25 \mathrm{eV}$ (harm.25).

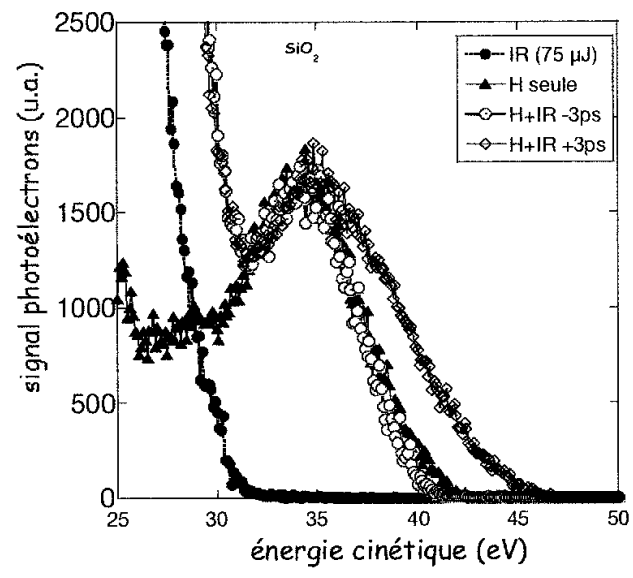

Figure2: spectres de photoémission obtenus sur le $\mathrm{SiO}_{2}$ avec le fuiscean harmonique de $39.25 \mathrm{eV}$ seul, le faisceal infrarouge seul( $1.57 \mathrm{eV}$ ) et les deux faisceanx avec la sonde IR arrivant 3 ps avant la pompe pais 3 ps après.

monocristaux $\mathrm{SiO}_{2}$ (quartz) et $\mathrm{Al}_{2} \mathrm{O}_{3}$, ceci n'est pas suffisant et une compensation de charge supplémentaire est réalisée au moyen d'un canon à électrons («flood gun »). La première étape expérimentale consiste à obtenir des spectres de photoémission corrects et reproductibles avec le faisceau harmonique. Par exemple sur la figure 1, le spectre de photoémission de $\mathrm{SiO}_{2}$ obtenu avec l'harmonique $25(\mathrm{~h} v=39.25 \mathrm{eV})$ est comparable à celui donné dans la littérature pour une excitation avec une lampe à hélium $(\mathrm{h} v=40.8 \mathrm{eV})$. On distingue deux pics séparés d'environ $5 \mathrm{eV}$, identifiés comme étant les niveaux électroniques des deux orbitales $2 p$ liantes et non liantes de l'oxygène [3]. Ensuite il s'agit d'observer un décalage du spectre qui indique que des électrons ont absorbé successivement un photon pompe et un photon sonde. Un tel effet pompe- sonde est clairement visible sur la figure 2 . Il se manifeste par un déplacement du spectre de photoélectrons vers les hautes énergies cinétiques lorsque la sonde arrive après l'impulsion harmonique. Pour des délais négatifssonde IR avant - l'absence de décalage indique que le spectre n'est pas perturbé par la charge d'espace ou par une mauvaise compensation de la charge de surface. A noter que pour une 
intensité IR plus élevée (fig.5 et 6), un décalage beaucoup plus important est obtenu, nous y reviendrons dans la conclusion.

Ce décalage dû à l'absorption d'un photon sonde diminue progressivement pour des retards croissants. Une mesure précise de cette évolution est obtenue en mesurant le signal à une énergie cinétique donnée en fonction du retard pompe- sonde (voir figures 3 et 4). On observe que la durée de vie des électrons est de l'ordre de quelques picosecondes à quelques dizaines de picosecondes, et d'autant plus longue que l'énergie cinétique des électrons considérés est basse. Ceci confirme les résultats obtenus précédemment sur le quartz avec l'harmonique 25 [4]. L'interprétation alors proposée de ce phénomène est que les électrons excités dans la bande de conduction perdent progressivement leur énergie cinétique par collisions électronsphonons, en même temps que la population diminue- diminution due aux pertes : émission à la surface, collisions inélastiques électrons- électrons, etc. Ce double processus de glissement vers les basses énergies et de baisse de population est bien illustré par les spectres pris à différents instants dans $\mathrm{CeF}_{3}$ : on observe en effet (fig.6) que le maximum de signal

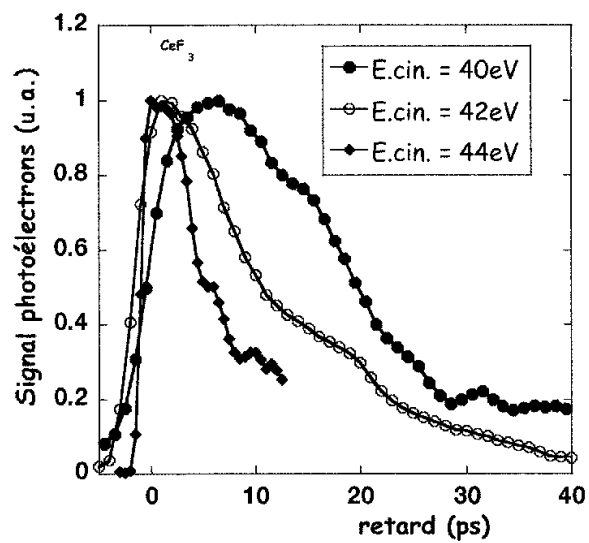

Figure 3: Pour le $\mathrm{CeF}_{3}$, signal de photólectrons en fonction du retard pompe sonde, pour 3 énergies cinétiques différentes $(40 \mathrm{eV}, 42 \mathrm{eV}$ et $44 \mathrm{eV}$ ) et une seule harmonique ( harm. 25).

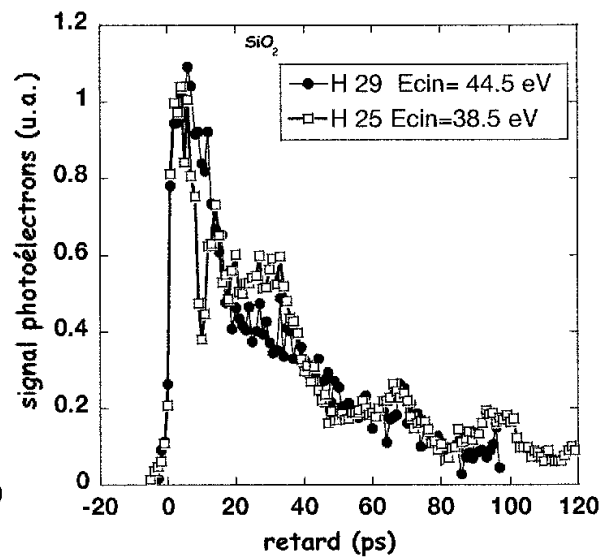

Figure 4: Pour le $\mathrm{SiO}_{2}$, signal de photoélectrons en fonction du retard pompe sonde, pour deux harmoniquess différentes $(H 25$ et H29)et deux énergies cinériques $(44.5$ eV et $38.5 \mathrm{eV})$.

initialement situé à $41 \mathrm{eV}$ se décale de $3 \mathrm{eV}$ après $10 \mathrm{ps}$, en même temps qu'il diminue. Cela se traduit sur les cinétiques (fig. 3) par un front de montée et une relaxation plus lents ainsi qu'un maximum retardé pour la cinétique mesurée pour des électrons de $40 \mathrm{eV}$ par rapport à ceux ayant une énergie de $44 \mathrm{eV}$. Une autre confirmation de cette hypothèse est donnée par la figure 4: en utilisant deux harmoniques différentes, on obtient la même cinétique de relaxation pour des photoélectrons ayant des énergies décalées d'une valeur égale à la différence d'énergie entre les deux harmoniques (i.e. $6 \mathrm{eV}$ ). A contrario, nous avons vérifié qu'une mesure à la même énergie cinétique pour deux harmoniques différentes donne des résultats différents. Cela signifie que dans les deux cas on sonde bien la relaxation de la même partie de la distribution des électrons excités par le photon harmonique.

\section{CONCLUSION}

Toutes les mesures que nous avons réalisées sur différents échantillons ( $\left.\mathrm{CsI}, \mathrm{Al}_{2} \mathrm{O}_{3}\right)$ et avec différentes harmoniques (dans la gamme 15 à 35) sont cohérentes et donnent des durées de vie de plusieurs picosecondes à dizaines de picosecondes, pour des électrons ayant des énergies cinétiques dans la bande de conduction de 15 à $45 \mathrm{eV}$. Des valeurs aussi élevées - de l'ordre 
de 100 ps - ont déjà été mesurées [5] pour des électrons de faible énergie dans le bas de la bande de conduction de diélectriques $\left(\mathrm{Al}_{2} \mathrm{O}_{3}, \mathrm{MgO}\right)$, mais elles sont a priori plus surprenantes dans cette gamme d'énergie où l'ionisation par impact est supposée être très efficace. Dans le cas de $\mathrm{SiO}_{2}$, la situation est particulièrement paradoxale. En effet la durée de vie des électrons excités en bas de bande de conduction par excitation multiphotonique de photons visibles ou
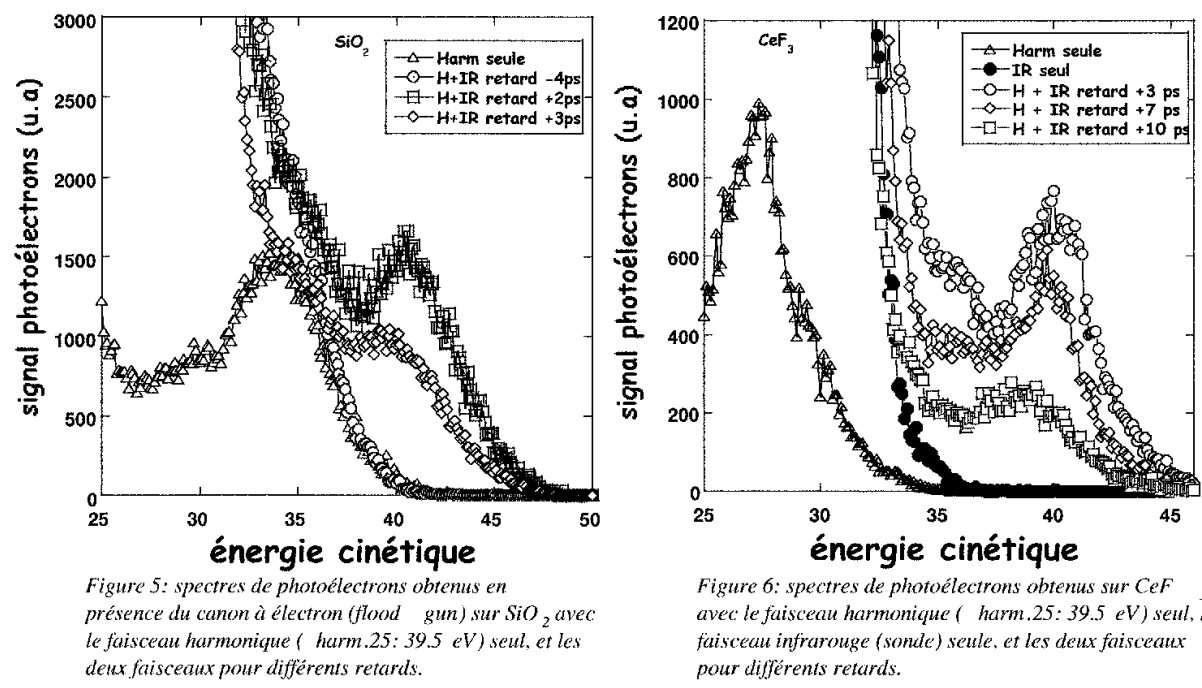

Figure 6: spectres de photoélectrons obtenus sur $\mathrm{CeF}$ avec le faisceau harmonicue ( harm.25: $39.5 \mathrm{eV}$ ) seul, le faiscea infrarouge (sonde) seule, er les deux faisceanx pour differents retards.

IR a été mesurée [5,6] et ces deux publications montrent que ces électrons de basse énergie sont piégés dans la bande interdite - sous forme d'excitons auto-piégés - après une demipicoseconde. On peut formuler différentes hypothèses pour expliquer ce résultat. En particulier, compte tenu de l'extrême sensibilité à la surface de la technique de photoémission, il est possible que nous sondions des états de surface plus faiblement couplés au réseau et perdant de ce fait moins vite leur énergie. Cependant ces hypothèses reposent sur le schéma simple d'absorptions successives d'un photon harmonique et d'un photon IR. Or celui-ci n'est pas suffisant pour rendre compte des décalages très importants (de $7 \mathrm{eV}$ dans $\mathrm{SiO}_{2}$ et $14 \mathrm{eV}$ dans $\mathrm{CeF}_{3}$, voir figures 5 et 6 ) des spectres observés à haute intensité sonde. L'absorption du nombre de photons IR (5 ou 9) correspondant à ces décalages est possible mais les processus d'ordres plus bas seraient dominants et conduiraient à une disparition de toute structure dans le spectre. Le mécanisme d'absorption d'énergie pour des intensités sonde importantes reste donc à identifier, par exemple en étudiant de façon plus systématique l'influence de l'intensité du faisceau sonde.

\section{REFERENCES}

[1] A. Rettenberger, P. Leiderer, M. Probst and R. Haight Phys. Rev. B 56 (1997) 12092.

[2] Bauer et al. , Phys. Rev. Lett. 87 (2001) 25501.

[3] Ibach H. and Rowe J.E. , Phys. Rev. B10 (1974) 710.

[4] F. Quéré, S. Guizard, Ph. Martin, and G. Petite. Phys. Rev. B 61, (2000), 9883.

[5] S. Guizard, P. Martin, P. Daguzan, G. Petite, P. Audebert, J.P. Geindre, A. Dos Santos, EuroPhys. Lett. 29 (1995) 401.

[6] P.N. Saeta and B. Greene, Phys. Rev. Lett. 70 (1993) 3588. 